\title{
Consenso de escoliosis idiopática del adolescente
} Adolescent idiopathic scoliosis

\author{
Comité Nacional de Adolescencia SAP, Comité de Diagnóstico por Imágenes SAP, \\ Sociedad Argentina de Ortopedia y Traumatología Infantil (SAOTI) y \\ Sociedad Argentina de Patología de la Columna Vertebral (SAPCV)
}

Comité Nacional de Adolescencia: Dra. M. Victoria Gacitúa ${ }^{a}$ Dra. María C. González Dr. Carlos Sanz $z^{b}$ Dra. Valeria Mullic, Dra. Patricia Goddard d, Dr. Eduardo D. Rolón ${ }^{e}$, Dra. Paulina Castellof, Dra. María del C. Hiebrag y Dra. Rosa Pappolla ${ }^{h}$

Comité de Diagnóstico por Imágenes: Dr. Luis F. Gentile Sociedad Argentina de Ortopedia y Traumatología Infantil (SAOTI) y Sociedad Argentina de Patología de la Columna Vertebral (SAPCV): Dr. Rubén Maenza

Colaboradores: Dr. Sergio Ruggiero, Dr. Alejandro Severini, Dra. Diana Pasqualini, Comité de Cuidados Críticos (secretario: Dr. Thomás Iolster; prosecretario: Dr. Guillermo Moreno; consultora: Dra. Silvia Santos; vocales titulares: Dra. Mariam I. Sarli, Dr. Guillermo Kohn Loncarica, Dr. Facundo Jorro Barón;

a. Hospital General de Agudos Dr. Cosme Argerich, Ciudad Autónoma de Buenos Aires. Servicio de Adolescencia.

b.Hospital de Niños Pedro de Elizalde, Ciudad Autónoma de Buenos Aires.

c. Hospital Italiano de Buenos Aires, Ciudad Autónoma de Buenos Aires.

d.Hospital Alemán,

Ciudad Autónoma de Buenos Aires.

e. CeSAC N. ${ }^{\circ}$ 35, Ciudad Autónoma de Buenos Aires.

f. Hospital Castro Rendón, Neuquén.

g. Hospital de Niños

Ricardo Gutiérrez,

Ciudad Autónoma de Buenos Aires.

h. Hospital José M. Penna, Ciudad Autónoma de Buenos Aires.

Correspondencia: Dra. M. Victoria Gacitúa, vickygacitua@gmail.com

Financiamiento:

Ninguno.

Conflicto de intereses: Ninguno que declarar.

Recibido: 4-7-2016

Aceptado: 12-7-2016 vocales suplentes: Dr. Daniel G. Buamscha, Dr. Guillermo L. Fagalde, Dr. Pablo Petracca), Comité de Medicina del Deporte Infanto Juvenil (secretaria: Dra. Daniela Pacheco Agrelo; prosecretaria: Dra. Laura V. Gaete; consultora: Dra. Elsa Galindo; vocales titulares: Dra. Maricel Sigle, Dra. Juliana M. R. Pochetti, Dr. Raúl Ferrari; vocales suplentes: Dr. Mario D. Ponczossznick, Dr. Leonardo M. Spada, Dra. Sandra Zagari; Dra. Nelly Testa, Dr. Marcelo Blanco, Dr. Juan Reviriego), Comité de Medicina Interna (secretario: Dr. Anibal A. Villa de Villafañe; prosecretario: Dr. Leonardo F. De Lillo; consultor: Dr. Néstor Abramovich; vocales titulares: Dra. Rosa M. Villasboas, Dr. Agustín Shaieb, Dra. María G. Gandolfi; vocales suplentes:

Dra. Claudia E. Sosa, Dra. Vanesa Zaslavsky, Dra. Gabriela Varone), Grupo de Trabajo de Kinesiología (secretaria: Lic. Martha Altina; integrantes:

Lic. M. Alejandra Timoni, Lic. Dardo Frachia, Lic. Gustavo Olguín, Lic. Judith Frydman, Lic. Judith Iglesias, Lic. Liliana Laspina, Lic. Pilar González, Lic. M. Laura Giménez, Lic. Mariana Pérez, Lic. Néstor Rosendo, Lic. Mariana Silva, Lic. Yamila Nocityc).

\section{RESUMEN}

La escoliosis es una condición patológica de la columna vertebral caracterizada por una deformidad espinal en las tres dimensiones del raquis (planos coronal, sagital y axial). Constituye una entidad de alta incidencia en la población pediátrica, especialmente en los adolescentes y en el sexo femenino.

Se caracteriza por la presencia radiográfica de una curva en el plano frontal, cuya magnitud es mayor de $10^{\circ}$ (técnica de Cobb). Puede ser idiopática o estar asociada a otras patologías. La escoliosis idiopática del adolescente (EIA) es aquella que se manifiesta entre los 10 años de edad y la madurez esquelética. Es más frecuente en mujeres $(70 \%)$. Su diagnóstico de "idiopática" es de exclusión, ya que su presencia también puede ser originada por anomalías neuroanatómicas de la fosa cerebral posterior o del conducto raquídeo.
En el examen físico, la presencia de giba/s visible/s con la maniobra de Adams (inclinación del tronco hacia adelante) expresa clínicamente la rotación vertebral.

Toda curva mayor de $20^{\circ}$ debe ser evaluada por el especialista. Las opciones de seguimiento y tratamiento son la observación, el tratamiento ortésico y la cirugía, dependiendo de la magnitud, de la progresión de la deformidad $\mathrm{y}$, fundamentalmente, de la clínica del paciente. Palabras clave: escoliosis, adolescente, maniobrade Adams, ángulo de Cobb, tratamiento.

\section{ABSTRACT}

Adolescent idiopathic scoliosis is a 3D spinal deformity in frontal, sagittal and axial planes, with high relevance in the pediatric population especially in adolescents and females between 10 years of age and the end of growth spurt and skeletal maturity. 
The radiographic manifestation is a curve greater than $10^{\circ}$ measured by Cobb method associated with vertebral rotation. "Idiopathic" diagnosis has to be done after neuroanatomical anomalies of the posterior cerebral fosa and spinal canal have been ruled out. The physical finding of a thoracic or lumbar hump is the clinical manifestation of vertebral rotation seen in a forward bending test (Adam's Test).

It is recommended that all curves with a magnitude greater than $20^{\circ}$ have to be controlled and treated by a spinal surgeon being observation, bracing and surgery the different treatment options based on the extent, progression of deformity and basically the clinical condition of the patient.

Key words: scoliosis, adolescent, Adam's test, Cobb's angle, treatment.

http://dx.doi.org/10.5546/aap.2016.585

\section{INTRODUCCIÓN}

La deformidad espinal constituye una condición de importancia diagnóstica en la población pediátrica, especialmente en la adolescencia, y puede ser un motivo de consulta o bien ser un hallazgo casual en el examen físico de rutina.

La escoliosis idiopática del adolescente (EIA) es la deformidad espinal más común. ${ }^{1}$ Está presente en el $2 \%-4 \%$ de los jóvenes entre 10 y 16 años. La gravedad de progresión es variable, desde aquellas que se autolimitan y no progresan a las que desarrollan formas progresivas $(10 \%)^{2}$ y/o graves, que pueden estar asociadas con otras anomalías orgánicas, sobre todo neurológicas.

El diagnóstico y el tratamiento precoz son de fundamental importancia. Es por ello por lo que su reconocimiento adecuado durante el control de salud es crucial para un tratamiento oportuno. ${ }^{3}$

\section{OBJETIVOS}

Resaltar la importancia de la pesquisa de la escoliosis en el examen físico y brindar las herramientas necesarias para su diagnóstico y tratamiento oportuno.

\section{METODOLOGÍA}

Se realizó una revisión bibliográfica, tanto a nivel nacional como internacional, de los últimos 15 años, mediante la consulta de las bases de Pubmed, Intramed, Cochrane y se consideraron opiniones de expertos nacionales.

\section{Definición}

La escoliosis es la desviación frontal del raquis asociada a rotación de los cuerpos vertebrales. La magnitud de la curva debe ser mayor de $10^{\circ}$ (técnica de Cobb) y asociada a rotación vertebral.
Ambas alteraciones deben ser visibles en la radiografía posteroanterior realizada con el paciente parado, descalzo y con el tronco bien relajado. ${ }^{4}$

La desviación de la columna con un valor angular $<10^{\circ}$ se conoce como asimetría espinal y no constituye una verdadera escoliosis.

Se denomina actitud escoliótica a aquellas desviaciones del raquis en el plano frontal que no presentan giba/s en el examen físico mediante la maniobra de Adams ni rotación radiográfica vertebral. Suelen ser posturales u originadas por discrepancia de los miembros inferiores (MM. II.). ${ }^{5}$

Tip: La escoliosis presenta giba/s en la maniobra de Adams y se observa en la radiografía con un ángulo de Cobb mayor de $10^{\circ}$. Si estos elementos faltan, no se considera una escoliosis verdadera.

\section{Clasificación}

Primaria o idiopática: Es la más frecuente $(80 \%)$, de causa desconocida, de origen multifactorial con componente genético. Existen tres categorías según la edad de aparición:

1. Infantil: Se manifiesta entre el nacimiento y los 3 años de edad. Afecta más a los varones y se relaciona con la posición supina de los bebés. El 70\%-90\% se resuelve espontáneamente.

2. Juvenil: Se manifiesta entre los 3 y los 10 años de edad. La incidencia es igual en niños y niñas, y sigue las reglas de progresión de la EIA.

3. Adolescente: Se manifiesta entre los 10 años de edad y la madurez esquelética. Es más frecuente en mujeres $(70 \%){ }^{6}$

Según su localización, puede ser cervical, torácica, torácica y lumbar (doble curva) o lumbar.

Los patrones más habituales, de acuerdo con la convexidad de la curva, son torácica derecha, doble curva torácica derecha y lumbar izquierda. ${ }^{7}$ Solo el $2 \%$ de las curvas torácicas son convexas a la izquierda y consideradas como patrón atípico de deformidad. ${ }^{8}$

Secundaria o sindrómica: Las escoliosis secundarias (20\%) están asociadas con distintas entidades (congénitas o adquiridas) (Tabla 1). ${ }^{2}$

\section{EVALUACIÓN DEL PACIENTE CON ESCOLIOSIS}

La EIA es un diagnóstico de exclusión. La historia clínica y el examen físico intentarán descartar otras causas secundarias de deformidad espinal. 


\section{Historia clínica}

1. Antecedentes familiares de deformidades de la columna o patología espinal.

2. Edad.

3. Estadio de madurez sexual de Tanner y edad de la menarca.

4. Dolor: La mayoría de las escoliosis en el adolescente son asintomáticas. Sin embargo, el $25 \%$ de los pacientes tiene dolor de espalda en la presentación inicial y otro 9\% lo desarrolla durante el seguimiento. Ante la presencia de dolor constante, nocturno o radicular, es necesario excluir una patología subyacente., ${ }^{910}$

5. Sintomas neurológicos: Disfunción vesical o anal, retraso madurativo motor o intelectual.

6. Antecedentes de enfermedades o traumatismos previos.

\section{Examen físico}

1. Talla: La determinación seriada de la talla ayuda a determinar el pico de empuje puberal (PEP), que constituye un buen factor de predicción relacionado con el grado de progresión de la curva. Para calcular el PEP, es necesario disponer de tallas en intervalos regulares de 6 meses, al menos, en tres oportunidades. En las niñas, este valor es de $9 \mathrm{~cm} /$ año y ocurre, en promedio, a los 12 años de edad cronológica $\mathrm{y}$, en los varones, es de $10 \mathrm{~cm} /$ año y ocurre, en promedio, a los 14 años.

2. Etapas de madurez sexual (estadios de Tanner), disponibles en http:/ / www.sap.org.ar/docs / publicaciones/libro_verde_sap_2013.

3. Piel y sistema músculo-esquelético: Manchas café con leche, nódulos subcutáneos y pecas axilares (neurofibromatosis de Von
Recklinghausen), parches pilosos lumbares $u$ hoyuelos en la piel lumbosacra (disrafismos) e hiperelasticidad (Marfan, Ehlers-Danlos).

4. Asimetría pelviana: Discrepancia de MM. II. (pseudoescoliosis).

5. Examen neurológico: La existencia de pies cavos, la debilidad en miembros superiores y/o inferiores, la asimetría o ausencia de los reflejos cutáneo-abdominales o la presencia de hiperreflexia rotuliana y/o aquileana deberían originar la sospecha del origen "no idiopático" de la escoliosis.

\section{Examen de la columna}

El paciente debe estar de pie, descalzo, con las piernas extendidas y la espalda descubierta y accesible al examinador con su postura habitual y sin correcciones.

Con el paciente de espalda, se debe observar lo siguiente (Figura 1):

- Nivel de los hombros.

- Nivel de las escápulas.

- Nivel de la pelvis.

- Centrado del tronco o signo de la plomada (debe coincidir entre la apófisis espinosa de la séptima vértebra cervical y la línea interglútea).

- Simetría del triángulo de la talla (relación entre el borde interno de los miembros superiores y del contorno del tronco).

- Maniobra de Adams: Se le solicita al paciente que se incline hacia adelante, con la cabeza lo más descendida posible sin flexionar las rodillas hasta que los hombros queden a la altura de las caderas mientras mantiene los brazos extendidos y péndulos y las palmas juntas (a modo de rezo). ${ }^{9-11}$

TABLA 1. Escoliosis secundaria

Escoliosis congénita

Escoliosis neuromuscular

Escoliosis sindromática

Otras causas
- Vertebral: mielomeningocele, hemivértebras, vértebras en cuña, barras vertebrales

- Extravertebral: fusiones congénitas de las costillas

- Formas neuropáticas: enfermedad de la motoneurona inferior (poliomielitis, mielomeningocele, trauma), enfermedad de la neurona motora superior (parálisis cerebral, traumatismos, tumores espinales, siringomielia)

- Formas miopáticas: progresiva (distrofia muscular), estática (artrogriposis)

- Síndromes mesenquimales: síndrome de Marfan, síndrome de Ehlers-Danlos

- Desórdenes metabólicos: osteogénesis imperfecta, raquitismo

- Neurofibromatosis

- Osteocondrodistrofias: enanismo acondroplásico, enanismo diastrófico, mucopolisacaridosis, displasia espondiloepifisaria

- Enfermedad reumatoidea

- Traumática (fractura, posirradiación, cirugía)

- Tumores óseos (osteoma osteoide, histiocitosis X)

Tabla 1. Causas de escoliosis secundaria. ${ }^{2}$ 
De esta manera, se evaluará la presencia de giba/s, expresión clínica de la rotación vertebral. La maniobra es positiva cuando la giba está presente (Adams positivo) y negativa cuando está ausente (Adams negativo). La maniobra detecta la rotación vertebral, pero no la cuantifica (Figura 2).

En una curva no estructurada (actitud escoliótica), no se evidencia giba (Adams negativo).

Medición del ángulo de rotación del tronco/ángulo de inclinación del tronco con escoliómetro:

El escoliómetro es un instrumento que se coloca en la espalda durante la maniobra de Adams y puede ser usado para brindar una medida objetiva de la rotación de la curvatura, pero su uso no es necesario para hacer el diagnóstico de escoliosis en atención primaria. ${ }^{8}$

La rotación de $5^{\circ}$ se extrapola $\left(\right.$ a $\left.20^{\circ}\right)(\mathrm{Cobb})^{2,10}$ (Figura 3).

- Maniobra de Adams sentado: Se utiliza para eliminar la oblicuidad de la pelvis en caso de asimetría de MM. II. Su especificidad aumenta porque elimina las asimetrías discretas debidas a discrepancia de longitud de MM. II., alteraciones de la cadera o distorsión pélvica. ${ }^{12}$
Con el paciente de perfil, se debe observar lo siguiente:

- Antepulsión de hombros.

- Abdomen prominente por falta de tono muscular abdominal.

FIgURA 2. Maniobra de Adams

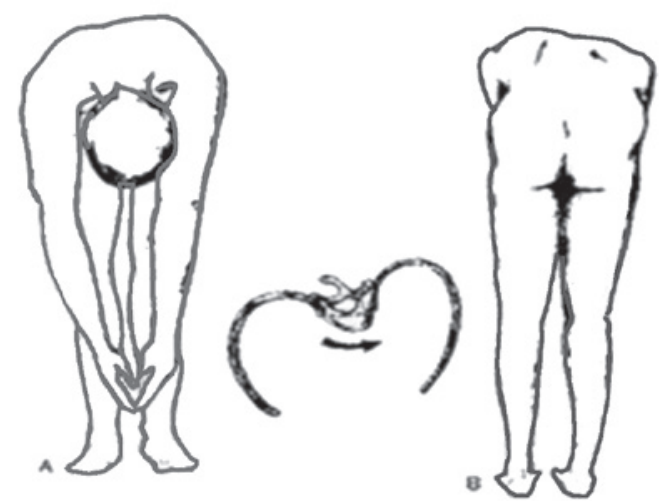

Maniobra de Adams: paciente inclinado hacia adelante, con la cabeza lo más descendida posible (mirando al piso) sin flexionar las rodillas hasta que los hombros queden a la altura de las caderas mientras mantiene los brazos péndulos y las palmas juntas (a modo de rezo). Se debe observar la asimetría de los hemidorsos, con la presencia de giba/s. La maniobra es positiva cuando la giba está presente (Adams positivo) y negativa cuando está ausente (Adams negativo).

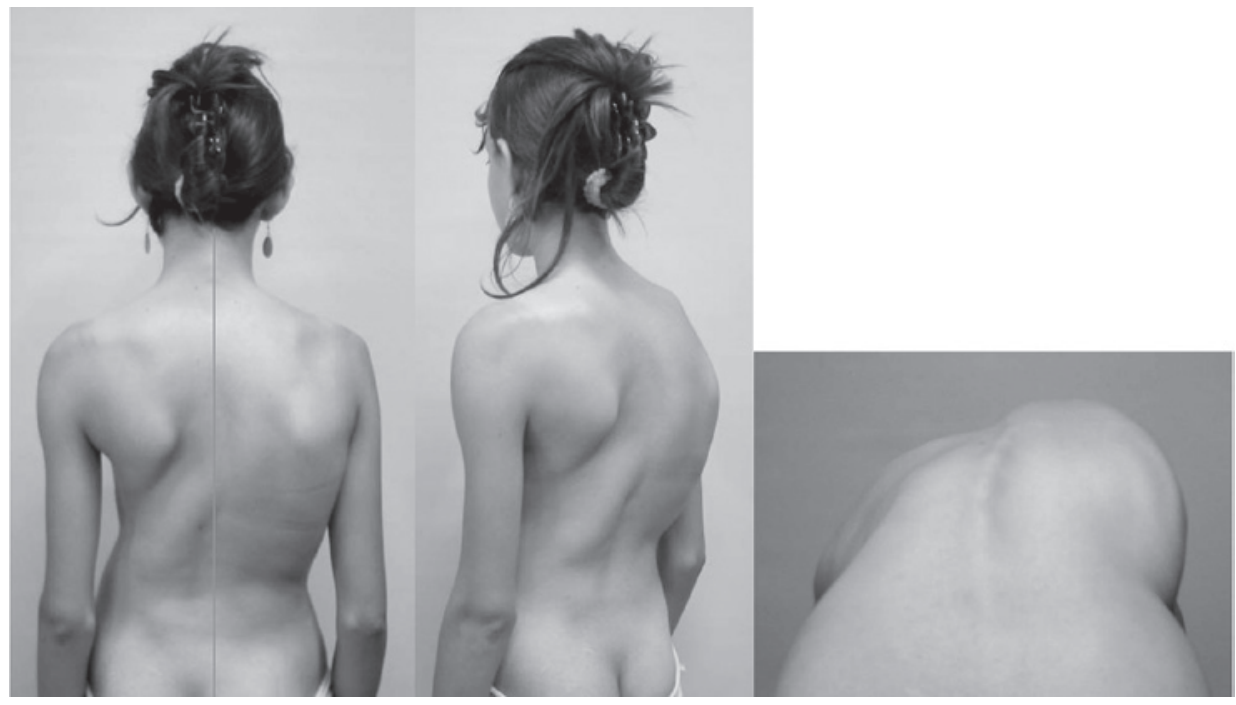

Examen físico del paciente portador de escoliosis. Aspectos clínicos en los planos frontal (la línea de la plomada -virtual desde C7- señala el tronco descentrado a la derecha), sagital y axial con la presencia de dos gibas importantes (torácica derecha y lumbar izquierda) con la paciente en inclinación hacia adelante (maniobra de Adams). (Cedida por el Dr. Maenza) 
Con el paciente de frente, se debe observar lo siguiente:

- Pectus excavatum o carinatum (Marfan)

- Simetría del desarrollo mamario

\section{DIAGNÓSTICO POR IMÁGENES}

El estudio radiológico debe ser solicitado ante la sospecha de escoliosis.

\section{Radiología simple}

Sigue siendo el método de elección para el diagnóstico y seguimiento de los pacientes con escoliosis.

El estudio radiológico inicial debe incluir totalmente la columna vertebral con cráneo y pelvis, espinograma de frente y perfil, con el paciente parado y descalzo, con el tronco bien relajado para evitar posiciones compensadoras. Los pacientes con dificultades para mantenerse en pie se explorarán en posición semisentada para mantener el efecto de la gravedad sobre las curvas.

\section{Evaluación de las radiografías:}

- Detectar defectos vertebrales congénitos: hemivértebras, barras óseas, escoliosis con patrón cervical, curvas cortas, hipercifosis.

- Identificar patrones de curvas de EIA: torácica, toracolumbar y/o lumbar. La curva se identifica según su convexidad en izquierda o derecha.

- Evaluar magnitud y extensión (Figura 4):

1.Identificación de las vértebras límites superior e inferior de la/s curva/s. El ápex se define como la región de la curva cuyas vértebras presentan mayor rotación y traslación en relación con la línea media. Es la zona más estructurada o menos flexible de la deformidad espinal. La extensión de la curva está limitada entre la vértebra límite superior (VLS) y la vértebra límite inferior (VLI). La VLS se define como la vértebra más proximal cuya carilla superior es la más inclinada al centro de la curva y la VLI es aquella cuya carilla inferior es la más inclinada al centro de la curva escoliótica.

2.Medición de la magnitud o valor angular. ${ }^{13}$ El ángulo de Cobb es el ángulo correspondiente y resultante del entrecruzamiento de las líneas perpendiculares a la carilla superior de la VLS y de la inferior de la VLI. Si las líneas no se cruzan, se trazan perpendiculares que se cruzarán y determinan el ángulo de la curva. Es anormal cuando es mayor de $10^{\circ}$. Toda curva mayor de $20^{\circ}$ debe ser evaluada por el especialista.

- Determinar la madurez esquelética: La inclusión de las crestas ilíacas permitirá medir la altura de la basculación de la pelvis y estimar el grado de madurez ósea mediante el signo de Risser (Figura 5). Este representa el estado de osificación de la cresta ilíaca, que comienza en la espina ilíaca anterosuperior (EIAS) y progresa hacia atrás en dirección a la espina ilíaca posterosuperior (EIPS): grado 0, sin osificación; 1: $25 \%$; 2 : $50 \%$; 3: $75 \%$; $100 \%$; y 5 : fusión con el ilíaco (osificación
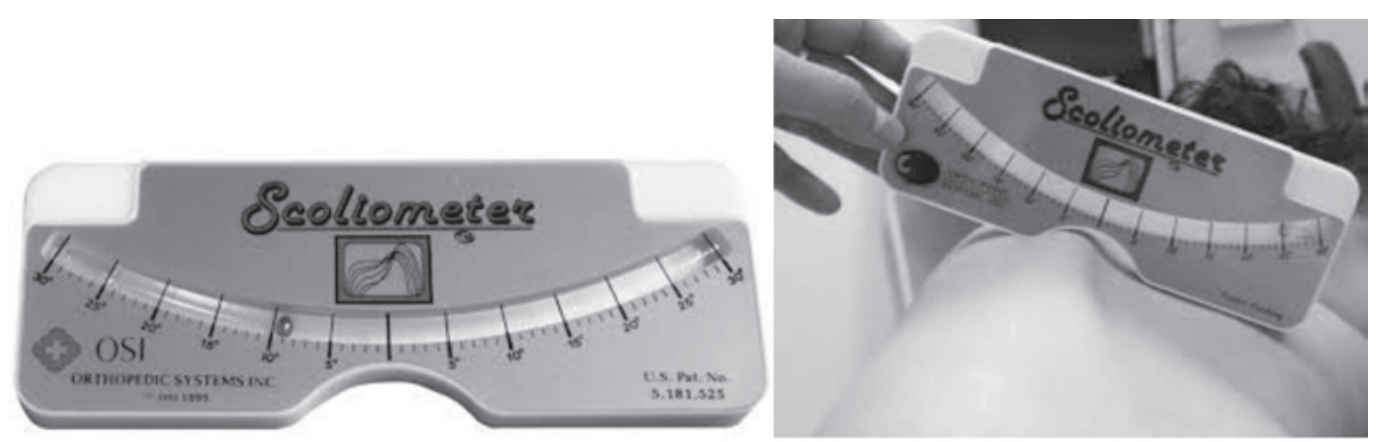

El escoliómetro es una forma efectiva y rápida de realizar la pesquisa escolar y mide la torsión del tronco en grados hacia ambos lados -bolilla-. Si señala $5^{\circ}$ de rotación -derecha o izquierda-, se extrapola a una desviación espinal radiográfica de $20^{\circ}$ de magnitud (Cobb) -derecha o izquierda- o más, por lo que se aconseja la derivación del paciente al especialista. Es la forma más rápida, menos costosa y más efectiva de realizar dichos controles, ya que no se necesita personal especializado para realizarlos. (Cedida por el Dr. Maenza) 
completa). Este último puede no coincidir con la detención del crecimiento vertebral. En caso de dudas sobre la madurez esquelética, puede solicitarse una radiografía de edad ósea.

El estándar para la evaluación de escoliosis es el espinograma (radiografía anteroposterior y lateral de pie en chasis largo de $30 \times 90 \mathrm{~cm}$ tomada a una distancia de $1,8 \mathrm{~m}$ ). No todos los centros cuentan con la posibilidad de realizar espinogramas y suele ser un estudio de alto costo. En pacientes con estatura menor de 1,60 m, podría reemplazarse por radiografía convencional de columna en chasis de $35 \times 43 \mathrm{~cm} .{ }^{10}$

Figura 4. Espinograma y medición de ángulo de Cobb

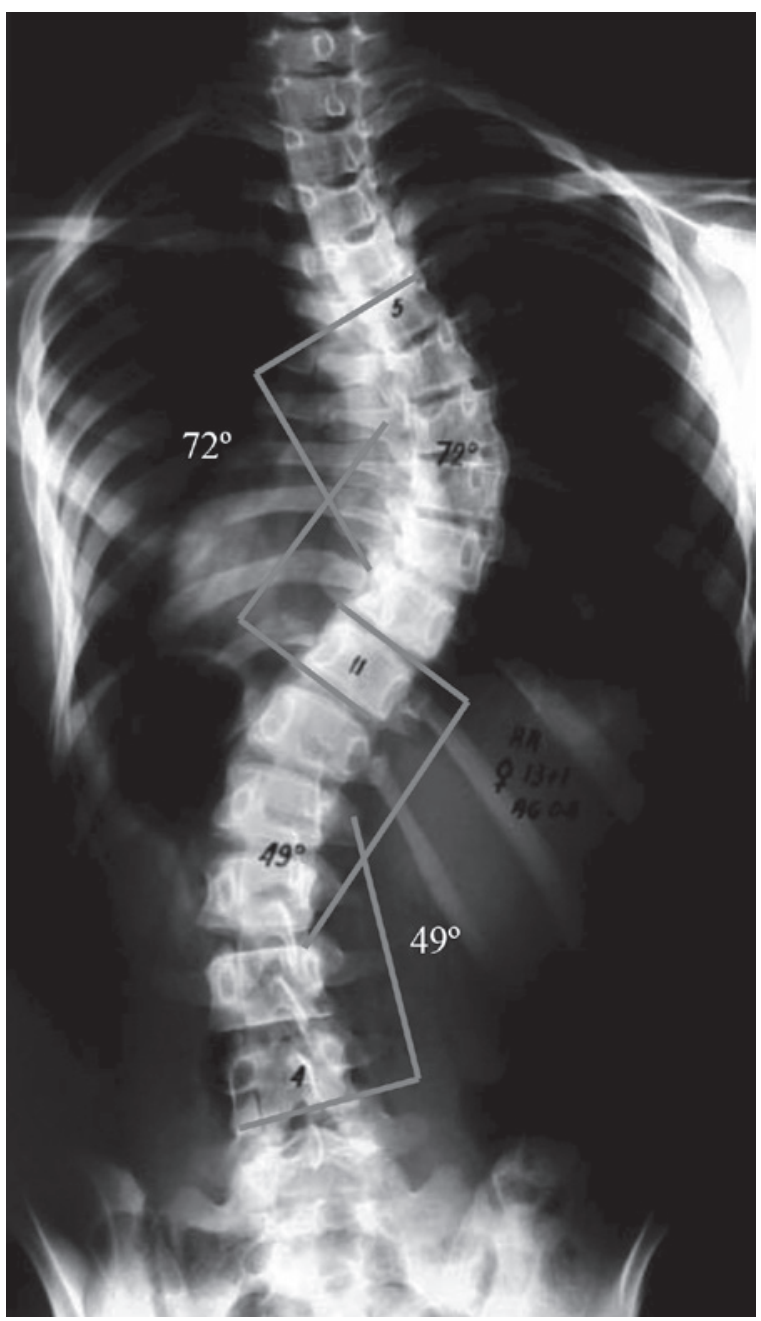

Espinograma que demuestra la magnitud angular (medición de Cobb) de una curva a doble patrón de deformidad torácica derecha-T5 (VLS), T11 (VLI)- y lumbar izquierda -T11 (VLS), L4 (VLI)-. (Cedida por el Dr. Maenza)

\section{Dosis de radiación}

Existe evidencia de que la exposición a la radiación ionizante aumenta el riesgo de mortalidad por cáncer de mama de las pacientes con escoliosis respecto a la población general. Teniendo en cuenta que un espinograma equivale a 100 radiografías de tórax, es necesario tomar medidas para reducir la dosis de radiación:

- Siempre que sea posible, se debe utilizar la proyección posteroanterior, que reduce la radiación mamaria y tiroidea hasta en un 70\%$80 \%{ }^{14}$

- Protección gonadal en ambos sexos.

- En los equipos analógicos, se sugiere utilizar protectores mamarios, innecesarios en los equipos digitales automáticos.

\section{Tomografía computarizada}

Es útil para definir mejor la anatomía en la evaluación de las anomalías vertebrales congénitas o ante la sospecha de causa tumoral. La tomografía computarizada (TC) sin contraste con reconstrucción en 3D es útil para planificar la cirugía. ${ }^{15}$ También debe tenerse en cuenta la radiación a la que se expone al paciente en cada tomografía (equivalente a 400 radiografías de tórax).

FIGURA 5. Evaluación de madurez esquelética: signo de Risser

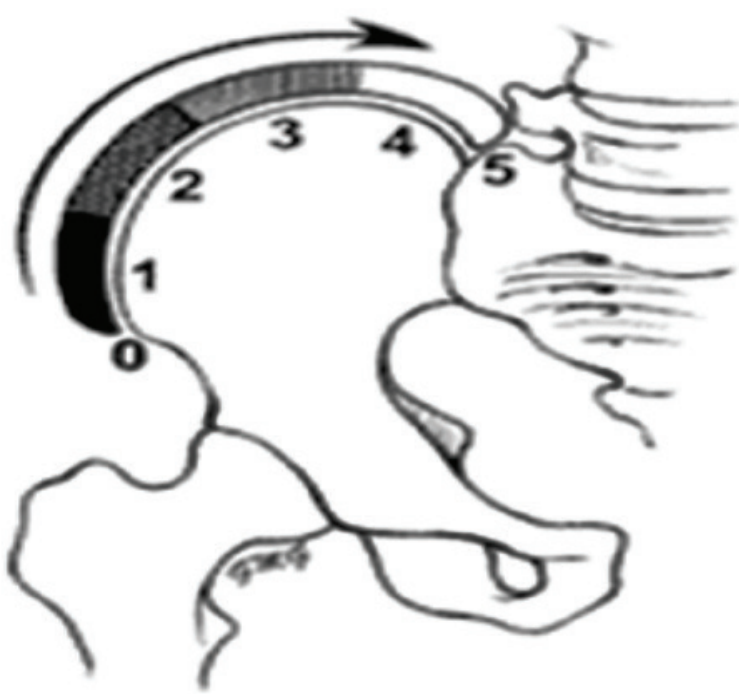

Estado de osificación de la cresta ilíaca, que comienza en la espina ilíaca anterosuperior y progresa hacia atrás en dirección a la espina ilíaca posterosuperior: grado 0 , sin osificación; 1: $25 \%$; 2: 50\%; 3: 75\%; 4: 100\%; y 5: fusión con el ilíaco. 


\section{Resonancia magnética}

Está indicada en los siguientes casos:

- Pacientes con escoliosis de inicio temprano (antes de los 11 años) con curvas mayores de $10^{\circ} .{ }^{6,17}$

- Presencia de dolor y/o rigidez.

- Pacientes con deformidad progresiva durante el tratamiento ortésico adecuado.

- Pacientes con patrón de curva atípica (torácica izquierda con o sin componente cervical). Pueden estar asociadas a causas secundarias.

- Curva de progresión rápida, sobre todo fuera del período activo de crecimiento puberal. ${ }^{16,17}$

- Pacientes con examen neurológico anormal y/o con antecedentes de cefaleas, cervicalgia y tortícolis.

- Pacientes con estigmas sindromáticos. ${ }^{3}$

- Antes del tratamiento quirúrgico de escoliosis para descartar cualquier anomalía del eje neural. ${ }^{17-20}$

\section{EVOLUCIÓN DE LA ESCOLIOSIS}

Una vez realizado el diagnóstico de escoliosis, es importante descartar la causa orgánica subyacente y determinar si la curva progresará. ${ }^{3}$

Esta información permite al médico diferenciar las curvas que requieren un cuidadoso monitoreo sistemático de las que requieren un tratamiento activo. $^{8}$

Los determinantes de progresión son la edad y el sexo del paciente, la etapa de desarrollo puberal, el crecimiento potencial, el patrón y el grado de curvatura al momento del diagnóstico. ${ }^{3}$ La combinación de estos factores puede ser útil para predecir casi el $89 \%$ de las curvas progresivas $^{10}$ (Tabla 2 ).

El crecimiento esquelético restante puede determinarse con la velocidad de crecimiento, con la edad de la telarca y la menarca, los estadios de maduración sexual de Tanner, el signo de Risser y/o la edad ósea. ${ }^{22}$ Se debe tener en cuenta que el PEP ocurre 6-12 meses antes de la menarca, en Tanner 3, promedio de 12 años en las niñas y, en los varones, en Tanner 4, promedio de 14 años $^{21}$ (Tabla 3).

Se debe revisar sistemáticamente la espalda de niños/as como parte del estudio rutinario y solicitar los estudios radiológicos correspondientes ante el hallazgo de giba en la maniobra de Adams.

\section{SEGUIMIENTO Y TRATAMIENTO}

Las opciones incluyen observación, corsé o cirugía.

TABLA 2. Factores de riesgo de progresión de la curva escoliótica ${ }^{10}$

\begin{tabular}{ll}
\hline Factor de riesgo de progresión & Comentario \\
\hline Edad & $\begin{array}{l}\text { A menor edad al momento del diagnóstico, mayor posibilidad de progresión durante el } \\
\text { crecimiento puberal. } \\
\text { Sexo }\end{array}$ \\
Menarca & La progresión posmenarca es menor. \\
Crecimiento esquelético restante & A mayor inmadurez esquelética, mayor riesgo de progresión. \\
Patrón de la curva & Las curvas dobles tienen mayor progresión. \\
Magnitud de la curva & Las curvas más graves progresan más rápido. \\
\hline
\end{tabular}

TABLA 3. Porcentaje de riesgo de progresión de la curva según valor angular de Cobb, sexo y edad ${ }^{8}$

\begin{tabular}{lcccc}
\hline & \multicolumn{4}{c}{ Edad al momento de la presentación } \\
\cline { 2 - 5 } Curva inicial en ${ }^{\circ}$ & Niñas de 10-12 años & Niñas de 13-15 años & Niñas > 15 años & Varones \\
\hline$<19$ & $25 \%$ & $1 \%$ & $<1 \%$ & $3 \%$ \\
$20-29$ & $60 \%$ & $40 \%$ & $10 \%$ & $6 \%$ \\
$30-56$ & $90 \%$ & $70 \%$ & $30 \%$ & \\
$>60$ & $100 \%$ & $90 \%$ & & \\
\hline
\end{tabular}


1. Según la madurez esquelética.

Inmadurez esquelética

- $<25^{\circ}$ : examen físico y radiografía cada 4-6 meses.

- $25^{\circ}-40^{\circ}$ : ortesis.

- $>40^{\circ}$ o progresión a pesar de la ortesis: se debe considerar la cirugía.

Madurez esquelética intermedia

- $<20^{\circ}$ : observación clínica.

- $20^{\circ}-30^{\circ}$ : radiografía cada 6-9 meses.

- $>30^{\circ}$ : radiografía cada 6 meses estrictamente.

- $>45^{\circ}$ : se debe considerar la cirugía.

Madurez esquelética

- $<40^{\circ}$ : sin especificar.

- $>40^{\circ}$ : evaluación anual.

- $\geq 50^{\circ}$ o curva en progresión: cirugía.

2. Según la gravedad de la curva (Tabla 4).

\section{TRATAMIENTO ORTÉSICO}

La Scoliosis Research Society (SRS) sugiere el tratamiento ortésico en las deformidades cuyas magnitudes superen los $25^{\circ}$ (medición de Cobb) en pacientes esqueléticamente inmaduros. El objetivo es evitar la progresión de la curva y no la corrección.

El corsé TLSO (toracolumbosacro-ortesis) representa esta forma de tratamiento. Existen otros tipos, como el corsé de Milwaukee.

La utilización del corsé -según la SRS- requiere un régimen de uso que depende de la situación clínica/radiográfica. Puede utilizarse el régimen de tiempo completo (20/22 horas/día) o de tiempo parcial (16/18 horas/día) durante la etapa de crecimiento activo puberal. Una vez finalizado este, se indicará el desuso paulatino del corsé con uso nocturno antes de la suspensión definitiva.

Un $20 \%$ de los casos aproximadamente pueden no responder a este tratamiento. Varios son los factores que dificultan el buen resultado: la carga genética, la presencia de anormalidades neuroanatómicas, la falta de adherencia del paciente por los problemas de imagen psicosocial y corporal. ${ }^{10}$

\section{ENFOQUE KINÉSICO}

Según la SRS, no existe evidencia científica que demuestre que los métodos de tratamiento kinésicos o manuales, tales como la manipulación, la estimulación eléctrica y el ejercicio corrector, detengan o mejoren la evolución de la escoliosis. ${ }^{23}$

Los ejercicios específicos para escoliosis (EEE) forman parte del enfoque kinésico. ${ }^{24}$ Tienen como objetivo trabajar sobre el tejido blando que se ve afectado por la disfunción de la columna vertebral para mantenerla flexible y mejorar la potencia muscular abdominal y paravertebral. No se reportaron efectos secundarios ni riesgos de su práctica.

La reeducación postural global (RPG) es un método novedoso fisioterapéutico que incluye ejercicios de flexibilización, deslordosantes y de armonización propioceptiva. La presentación de casos individuales de escoliosis en las publicaciones periódicas de las diferentes asociaciones en la disciplina está demostrando resultados favorables, si bien no existen ensayos clínicos que evalúen su efectividad. ${ }^{25}$

\section{TRATAMIENTO QUIRÚRGICO}

Para los adolescentes con una curva con un ángulo de $\mathrm{Cobb}>45^{\circ}-50^{\circ}$, se recomienda la cirugía. Casi el 10\% de los adolescentes con escoliosis idiopática sufrirá la progresión de la escoliosis hasta requerirla.

La decisión de proceder a la corrección quirúrgica requiere considerar fundamentalmente la evaluación clínica, la presencia de comorbilidades asociadas y los deseos del paciente en cuanto al impacto que produce su condición clínica en relación con su calidad de vida desde el punto de vista psicosocial.

El objetivo de la cirugía es corregir la deformidad ya instalada y mejorar las condiciones estéticas para evitar así las potenciales alteraciones funcionales cardiorrespiratorias que suelen ocurrir cuando las curvas alcanzan magnitudes mayores de $110^{\circ}-120^{\circ}$.

TABLA 4. Opciones de tratamiento según la gravedad de la curva expresada en grados de Cobb ${ }^{8}$

\begin{tabular}{ll}
\hline Curva en $^{\circ}$ & Terapia \\
\hline $0-25^{\circ}$ & Observación seriada ante inmadurez esquelética \\
$25-30^{\circ}$ con progresión de $5-10^{\circ}$ & Corsé \\
$30-40^{\circ}$ & Corsé \\
Mayor de $40^{\circ}$ & Cirugía en pacientes esqueléticamente inmaduros \\
Mayor de $50^{\circ}$ & Cirugía en pacientes esqueléticamente maduros \\
\hline
\end{tabular}


El abordaje quirúrgico más común de la EIA es el posterior, a través de una incisión longitudinal en la línea media posterior. Se insertan tornillos transpediculares en los segmentos vertebrales asociados a dos barras de metal (actualmente, de cromo cobalto) contorneadas.

El ajuste de estas varillas a los tornillos y ciertas maniobras quirúrgicas, como la desrotación de las barras y de la compresión y distracción de cada uno de ellos, permiten la corrección de la deformidad espinal. La instrumentación espinal siempre está asociada a la colocación de injerto óseo del paciente (injerto autólogo) con el objeto de obtener la fusión o artrodesis definitiva, proceso del que, en último lugar, dependerá el mantenimiento de la corrección de la deformidad espinal obtenida quirúrgicamente.

La recuperación puede llevarse a cabo en Sala de Internación Pediátrica o en la Unidad de Cuidados Intensivos Pediátricos, según los recursos institucionales. En ambos casos, es indispensable que se cumpla una adecuada relación enfermero-paciente (1:1 o 1:2) con personal entrenado para cumplir con los controles necesarios. ${ }^{26}$

El objetivo de los controles durante el período posquirúrgico inmediato es la detección de las posibles complicaciones, el adecuado monitoreo y el tratamiento del dolor con opiáceos.

Las complicaciones inherentes a este tipo de cirugía son las siguientes:

- Clínicas: neumotórax, atelectasias, íleo, hidrotórax, hemorragia, síndrome de secreción inapropiada de hormona antidiurética.

- Infecciosas: pueden ocurrir en el posoperatorio inmediato o tardío. Su incidencia es del 2\%-4\%. En algunos centros, se realiza la búsqueda de Staphylococcus aureus meticilino resistente (SAMR) en cultivos nasales, axilares e inguinales antes de la cirugía, y, de ser positivos, se realiza descontaminación con jabón de clorhexidina y mupirocina nasal. ${ }^{27}$ Se recomienda, actualmente, la profilaxis prequirúrgica con vancomicina y ceftazidima/ cefazolina y el lavado pulsátil como técnica de irrigación intraoperatoria. Estas dos medidas han demostrado ser efectivas en reducir las infecciones posquirúrgicas. ${ }^{28}$ La profilaxis antibiótica debe mantenerse durante las primeras $24 \mathrm{~h}$. Este esquema puede modificarse según epidemiología y protocolo de cada institución.

- Neurológicas: constituyen la complicación más grave. Pueden ser lesiones medulares o radiculares completas o incompletas, transitorias o definitivas. Su incidencia es del 0,7\%, según el Comité de Morbimortalidad 2012 de la SRS, en 11000 cirugías de EIA reportadas en dicho año. Debido a los avances en la técnica quirúrgica y la tecnología, estas complicaciones son cada vez menos frecuentes. La corrección quirúrgica de la escoliosis debe realizarse bajo monitoreo neurofisiológico intraquirúrgico con potenciales evocados somatosensitivos y motores para evitar lesiones medulares..$^{29-31}$

- Pseudoartrosis: es la falta de consolidación e incorporación del injerto óseo, que puede ocasionar ruptura del implante espinal y necesidad de revisión quirúrgica. Su incidencia es desconocida por su difícil detección clínica y/o imagenológica.

\section{ESCOLIOSIS Y DEPORTE}

Si bien la evidencia actual no ofrece pruebas de que se pueda alterar la evolución natural de la escoliosis mediante ejercicios, existen efectos beneficiosos generales de la terapia por ejercicios, como así también de las actividades deportivas sobre la flexibilidad y movilidad del raquis, la fuerza muscular, la función pulmonar, la capacidad aeróbica, el equilibrio y la propiocepción, entre otros, que pueden ser de utilidad.

Además de estos beneficios funcionales, la práctica deportiva confiere beneficios adicionales, como la mejora de la autoestima y la socialización, que, a menudo, se ven afectadas en pacientes con

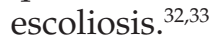

No existe evidencia científica para pensar que actividades deportivas de ningún tipo aumenten el riesgo de progresión de la escoliosis, por lo tanto, es un error desaconsejar la práctica deportiva.

La mayoría de los pacientes con EIA puede participar normalmente en deportes y en actividades recreativas e, incluso, realizar deportes asimétricos, que ocasionan hipertrofia muscular del lado dominante y pueden dar la impresión de una alteración del eje del tronco (tenis, hockey, lanzamientos, esgrima, etc.), con el monitoreo cuidadoso por el especialista. ${ }^{34}$

Los pacientes que han recibido tratamiento quirúrgico de la escoliosis pueden tener restricciones para realizar actividad física vigorosa con alto impacto sobre la columna vertebral; en estos casos, se recomienda individualizar las indicaciones con el especialista. ${ }^{35}$ 


\section{CONCLUSIONES}

El examen de la columna debe realizarse como parte del control clínico anual con especial atención en la preadolescencia y la adolescencia.

El adolescente con EIA puede ser seguido por su médico de cabecera si la curva presenta un bajo riesgo de progresión y se ha descartado patología subyacente.

Deben ser derivados al especialista dedicado a la cirugía espinal pediátrica aquellos adolescentes que presentan los siguientes:

- Curvas progresivas,

- Inmadurez esquelética y alto riesgo de progresión,

- Curvas atípicas,

- Compromiso neurológico,

- Dolor o rigidez,

- Estigmas sindromáticos.

\section{Agradecimientos}

Comité de Pediatría Ambulatoria y Grupo de Trabajo de Salud Escolar.

\section{REFERENCIAS}

1. Chin K, Price JS, Zimbler S. A guide to early detection of scoliosis. Contemp Pediatr 2001;18(9):77.

2. Reamy BV,SlakeyJB. Adolescent idiopathic scoliosis: review and current concepts. Am Fam Physician 2001;64(1):111-6.

3. Hresko MT. Clinical practice. Idiopathic scoliosis in adolescents. N Engl J Med 2013;368(9):834-41.

4. Morrissy RT, Weinstein SL. Lovell and Winter's pediatric orthopaedics. $6^{\text {th }}$ ed. Philadelphia: Lippincott Williams \& Wilkins; 2006.Págs.693-762.

5. Noguera G. Problemas ortopédicos comunes. Parte 2. Comunicación personal.

6. Chorley JN, Neinstein LS. Scoliosis and kyphosis. En: Neinstein LS, ed. Adolescent Health Care: a practical guide. $4^{\text {th }}$ ed. Philadelphia: Lippincott Williams \& Wilkins; 2002. Págs.369-82.

7. Groiso JA. Patología ortopédica. En Sociedad Argentina de Pediatría, ed. Atención integral de adolescentes y jóvenes. Criterios de diagnóstico y tratamiento. Volumen 2. Buenos Aires: Sociedad Argentina de Pediatría; 1996.Págs.305-18.

8. BuecheMJ.Scoliosis and kyphosis. En:Neinstein LS, Gordon CM,Katzman DK, Rosen D, etal, eds. Adolescent Health Care: a practical guide. $5^{\text {th }}$ ed. Philadelphia: Lippincott Williams \& Wilkins; 2007.Págs.235-42.

9. Janicki JA, Alman B. Scoliosis: Review of diagnosis and treatment. Paediatr Child Health 2007;12(9):771-6.

10. Altaf F, Gibson A, Dannawi Z, Noordeen H. Adolescent idiopathic scoliosis. BMJ 2013;346:f2508.

11. Yufra D, Giordana G. Escoliosis idiopática del adolescente en la provincia de Jujuy chequeo selectivo 2007-2009. Rev Asoc Argent Ortop Traumatol 2011;76(3):211-23.

12. KotwickiT,NegriniS, Grivas TB, Rigo M, etal. Methodology of evaluation of morphology of the spine and the trunk in idiopathic scoliosis and other spinal deformities - 6th SOSORT consensus paper. Scoliosis 2009;4:26.

13. Vallejos Meana N, Rositto V, Legarreta C, Escalada M, et al. Evaluación por imágenes de deformidades de la columna vertebral. Arch Argent Pediatr 2003;101(6):501-6.

14. Winter RB, Lonstein JE, Heithoff KB, Kirkham JA. Magnetic resonance imaging evaluation of the adolescent patient with idiopathic scoliosis before spinal instrumentation and fusion. A prospective, double-blinded study of 140 patients. Spine (Phila Pa 1976) 1997;22(8):855-8.
15. Lonegro SL. Indicación racional de estudios por imágenes. PRONAP 2014. Módulo2. Buenos Aires:Sociedad Argentina de Pediatría; 2014.Págs.13-30.

16. Charry O, KoopS, Winter R, Lonstein J, et al. Syringomyelia and scoliosis: a review of twenty-five pediatric patients. J Pediatr Orthop 1994;14(3):309-17.

17. Tello CA, Bersusky E, Francheri Wilson A. Escoliosis, siringomielia y malformación de Arnold-Chiari: las alteraciones espinales como manifestación inicial de estas últimas enfermedades. Rev Asoc Argent Ortop Traumatol 1991;56(2):236-49.

18. Huebert HT, MacKinnon WB. Syringomyelia and scoliosis. J Bone Joint Surg Br 1969;51(2):338-43.

19. Nordwall A, Wikkelao C. A late neurologic complication in scoliosis surgery in connection with syringomyelia. Acta Orthop Scand 1979;50(4):407-10.

20. RiseboroughEJ,HerndonJH.Scoliosis and other deformities of the axial skeleton. Boston: Little Brown: 1975.

21. Sanders JO. Maturity indicators in spinal deformity. J Bone Joint Surg Am 2007;89(Suppl 1):14-20.

22. Knott P, Pappo E, Cameron M, Demauroy J, et al. SOSORT 2012 consensus paper: reducing $x$-ray exposure in pediatric patients with scoliosis. Scoliosis 2014;9:4.

23. Pantoja S, Chamorro M. Escoliosis en niños y adolescentes. Rev Med Clin Condes 2015;26(1):99-108.

24. Romano M, Minozzi S, Bettany-Saltikov J, Zaina F, et al. Exercises for adolescent idiopathic scoliosis. Cochrane Database Syst Rev 2012;(8):CD007837.

25. Aristegui Racero G. Eficacia del tratamiento de Reeducación Postural Global (RPG) en adolescentes con Escoliosis Idiopática Combinada atendidos con una frecuencia de una vez por semana [Internet]. [Acceso: 12 de julio de 2016]. Disponible en: http:/ / www.sinergia-web.com/archivos/ RPGenescoliosis.pdf.

26. Shan LQ, Skaggs DL, Lee C, Kissinger C, et al. Intensive care unit versus hospital floor: a comparative study of postoperative management of patients with adolescent idiopathic scoliosis. J Bone Joint Surg Am 2013;95(7):e40.

27. Glotzbecker MP, Riedel MD, Vitale MG, Matsumoto $H$, et al. What's the evidence? Systematic literature reviews of risk factors and preventive strategies for surgical site infection following pediatric spine surgery. J Pediatr Orthop 2013;33(5):479-87.

28. Myung KS, Glassman DM, Tolo VT, Skaggs DL. Simple steps to minimize spine infections in adolescent idiopathic scoliosis. J Pediatr Orthop 2014;34(1):29-33.

29. Stecker MM. A review of intraoperative monitoring for spinal surgery. Surg Neurol Int 2012;3(Suppl 3):S174-87.

30. Azabou E, Manel V, Abelin-Genevois K, Andre-Obadia $\mathrm{N}$, et al. Predicting intraoperative feasibility of combined TES-mMEP and cSSEP monitoring during scoliosis surgery based on preoperative neurophysiological assessment. Spine J 2014;14(7):1214-20.

31. Nuwer MR, Emerson RG, Galloway G, Legatt AD, et al. Evidence-based guideline update: intraoperative spinal monitoring with somatosensory and transcranial electrical motor evoked potentials: report of the Therapeutics and Technology Assessment Subcommittee of the American Academy of Neurology and the American Clinical Neurophysiology Society. Neurology 2012;78(8):585-9.

32. San Segundo-Mozo R, Valdés-Vilches M, Aguilar-Naranjo JJ. Tratamiento conservador de la escoliosis. Papel de la cinesiterapia. Rehabilitacion (Madr) 2009;43(6):281-6.

33. Mordecai SC, Dabke HV. Efficacy of exercise therapy for the treatment of adolescent idiopathic scoliosis: a review of the literature. Eur Spine J 2012;21(3):382-9.

34. GielenJL, Van den EedeE. Scoliosis and sports participation. Internat Sport Med J 2008;9(3):131-40.

35. Weinstein SL, Dolan LA, Spratt KF, Peterson KK, et al. Health and function of patients with untreated scoliosis: a 50-year natural history. JAMA 2003;289(5):559-67. 